\title{
Transversity signals at COMPASS
}

\author{
Federica Sozzi ${ }^{\mathrm{a}}$ (On behalf of the COMPASS collaboration) \\ aTrieste University and INFN Trieste, \\ via Valerio, 2, 34127 Trieste, Italy \\ federica.sozzi@ts.infn.it
}

COMPASS is a fixed target experiment at the CERN SPS, with a rich physics program focused on nucleon spin structure and on hadron spectroscopy. One of the main goals of the spin program is the measurement of the transverse spin distribution function $\Delta_{T} q(x)$ in semi-inclusive DIS off transversely polarized nucleons. For this purpose approximately $20 \%$ of the running time in the years 2002 to 2004 with the longitudinally polarized muon beam of $160 \mathrm{GeV}$ and with ${ }^{6} \mathrm{LiD}$ polarized target was used to collectdata with the target polarized transversely with respect to the beam direction. The 2002 data have been already analysed and published. We present here the preliminary results from the full statistics for the Collins and Sivers single hadron asymmetries and for the transverse spin asymmetry in hadron pair production.

\section{INTRODUCTION}

The nucleon spin structure can be described at leading twist by three parton distribution functions [1]: the unpolarized distribution $q(x)$, the helicity distribution $\Delta q(x)$ and the transversity distribution $\Delta_{T} q(x)$, describing the probability in a transversely polarized nucleon of finding a quark with spin parallel to the nucleon spin.

The $\Delta_{T} q(x)$ distribution is still largely unknown, and this is the reason why transversity nowadays has an important role in the scientific programme of different experiments: HERMES at DESY, RHIC at BNL and COMPASS at CERN.

The COMPASS collaboration is measuring transversity in semi-inclusive DIS (SIDIS) of leptons on transversely polarized nucleons, using different quark polarimeters: azimuthal distribution of single hadrons, azimuthal dependence of the plane containing hadron pairs, and measurement of transverse polarization of baryons ( $\Lambda$ hyperons).

In this contribution, we will present results on single hadron and hadron pair asymmetries.

\section{SINGLE HADRON ASYMMETRIES}

Due to its chiral odd nature, in SIDIS the $\Delta_{T} q(x)$ function can be assessed only together with another chiral odd function.

One possibility is to measure it together with the so called Collins function $\Delta_{T}^{0} D_{q}^{h}[2]$. According to Collins, the fragmentation of a transversely polarized quark into an unpolarized hadron has an azimuthal dependence, with respect to the plane defined by the quark momentum and the quark spin. For this reason the event yield can be written as:

$N=N_{0} \cdot\left(1+f \cdot P_{t} \cdot D_{n n} \cdot A_{C} \cdot \sin \left(\phi_{C}\right)\right)$

where $f$ is the target dilution factor, $P_{t}$ the target polarization and $D_{n n}=(1-y) /\left(1-y+y^{2} / 2\right)$ the transverse spin transfer coefficient from the initial to the struck quark. The Collins angle $\phi_{C}$ is defined as $\phi_{h}-\phi_{s^{\prime}}$, where $\phi_{h}$ is the angle of the transverse momentum of the outgoing hadron and $\phi_{s^{\prime}}=\pi-\phi_{s}$ is the azimuthal angle of the struck quark spin $\left(\phi_{s}\right.$ is the azimuthal angle of quark before the hard scattering). $A_{C}$ is the Collins asymmetry coming from the coupling between the Collins fragmentation function and the transverse spin distribution:

$A_{C}=\frac{\sum_{q} e_{q}^{2} \Delta_{T} q(x) \Delta_{T}^{0} D_{q}^{h}\left(z, p_{T}^{2}\right)}{\sum_{q} e_{q}^{2} q(x) D_{q}^{h}\left(z, p_{T}^{2}\right)}$

where $z=E_{h} /\left(E_{l}-E_{l^{\prime}}\right)$ is the fraction of available energy carried by the hadron, and $p_{T}$ is the hadron transverse momentum with respect to the virtual photon direction. 
As is clear from eq1 the Collins asymmetry $A_{C}$ is revealed as a $\sin \phi_{C}$ modulation in the number of produced hadrons.

Another azimuthal asymmetry is related to the Sivers effect [3], arising from a possible coupling of the intrinsic transverse momentum $k_{T}$ of unpolarized quarks to the spin of a transversely polarized nucleon. In this case the dependence of the number of produced hadrons is:

$N=N_{0} \cdot\left(1+f \cdot P_{t} \cdot A_{S} \cdot \sin \left(\phi_{S}\right)\right)$

where the Sivers angle $\phi_{S}$ is defined as $\phi_{h}-\phi_{s}$, and the asymmetry $A_{S}$ probes the so called Sivers distribution function, $\Delta_{0}^{T} q$ :

$A_{S}=\frac{\sum_{q} e_{q}^{2} \Delta_{0}^{T} q\left(x, k_{T}\right) D_{q}^{h}(z)}{\sum_{q} e_{q}^{2} q(x) D_{q}^{h}(z)}$.

In this case the asymmetry $A_{S}$ is revealed as a $\sin \phi_{S}$ modulation in the number of produced hadrons. It has to be noted here that since the Collins and the Sivers angles are independent 4, it is possible to measure from the same data both the Collins and the Sivers effects.

\subsection{RESULTS}

We present the recent results for Collins and Sivers asymmetries on all the data available up to now.

The results from the first physics run we had in 2002 have already been published [5], while another paper containing all our statistics (20022004 data taking) is going to be published 6 .

The COMPASS experiment [7] [8] uses a longitudinally polarized $\mu^{+}$beam of $160 \mathrm{GeV} / \mathrm{c}$ and a two cells ${ }^{6} \mathrm{LiD}$ target, whose nucleons are polarized transversely respect to the beam direction. The ${ }^{6} \mathrm{LiD}$ material is characterized by a high dilution factor $f \sim 0.38$, and the target polarization is about $50 \%$. The nucleons in the two target cells (upstream and downstream cell) have opposite polarizations to minimize systematic effects; the polarizations are reversed once per week. The asymmetries are extracted using at the same time the information coming from both cells in two periods with opposite configuration; this is done building the estimator:

$A_{j}\left(\phi_{j}\right)=\frac{N_{j, u}^{\uparrow}\left(\phi_{j}\right)}{N_{j, u}^{\downarrow}\left(\phi_{j}\right)} \cdot \frac{N_{j, d}^{\uparrow}\left(\phi_{j}\right)}{N_{j, d}^{\downarrow}\left(\phi_{j}\right)}, \quad j=C, S$ and fitting the measured $A_{j}\left(\phi_{j}\right)$ values with the functions $p_{0} \cdot\left(1 .+A_{C}^{r a w} \cdot \sin \phi_{C}\right)$ and $p_{0} \cdot(1 .+$ $\left.A_{S}^{r a w} \cdot \sin \phi_{S}\right)$, where $p_{0}$ is a free parameter. The physical asymmetries are extracted from the raw asymmetries $A_{C}^{r a w}$ and $A_{S}^{r a w}$ knowing f, $P_{T}$ and also $D_{n n}$ in the Collins case.

In the analysis, the events are considered only if one primary vertex with incoming and outgoing $\mu$ is found in the target region. To select DIS events, the photon virtuality $Q^{2}$ is taken above $1(\mathrm{GeV} / \mathrm{c})^{2}$, the fractional energy of the virtual photon $y$ between 0.1 and 0.9 , and the invariant mass of the final hadronic state $W$ above 5 $\mathrm{GeV} / \mathrm{c}^{2}$.

The hadron sample on which the asymmetries are computed consist of all the hadrons coming from the reaction vertex with $p_{T}>0.1 \mathrm{GeV} / \mathrm{c}$ and $z>0.2$. Also a leading hadron sample is considered, using the most energetic hadron with $z>0.25$ for each event.

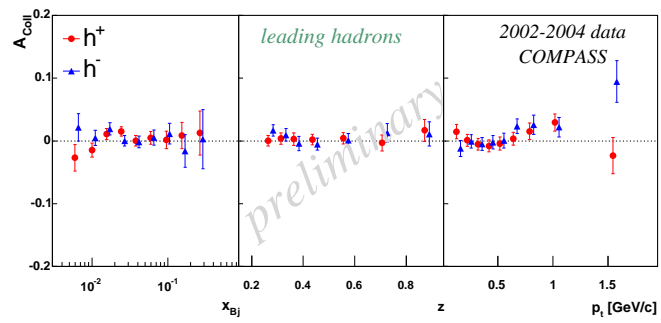

Figure 1. Collins asymmetries for positive (circles) and negative (triangles) leading hadrons as a function of $x, z$ and $p_{T}$.

The results on the Collins and Sivers asymmetries, plotted as a function of the kinematical variables $x, z$ and $p_{t}$, are shown in fig. 1 and 2 for leading hadrons. A lot of work has been done in order to investigate possible sources of systematic effects; the conclusion of all the studies is that the systematic error is considerably smaller than the statistical one, shown in the plots.

All the asymmetries measured are small and 


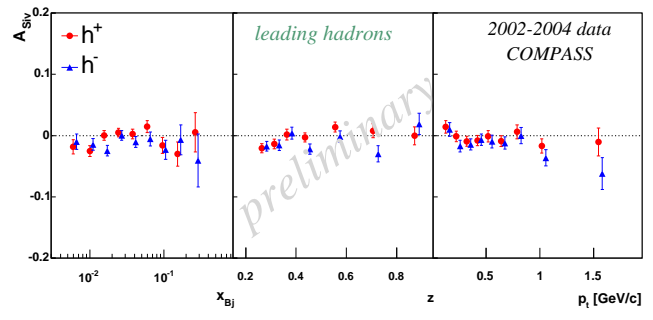

Figure 2. Sivers asymmetries for positive (circles) and negative (triangles) leading hadrons as a function of $x, z$ and $p_{T}$.

compatible with zero within the quoted errors. The result is compatible with some phenomenological works suggesting the cancellation between the $\mathrm{p}$ and $\mathrm{n}$ contribution in an isoscalar target as the one used by COMPASS.

Results on identified hadrons have recently been produced. The hadron identification is done using the RICH-1 detector 9 in the COMPASS spectrometer.

Before applying RICH-1 in the analysis, several studies have been performed in order to ensure the detector stability throught time: the number of hadron identified as $\pi$ and $K$ normalized to the number of reconstructed tracks have been monitored in time. The runs or spills were rejected in correspondance of a deviation larger than 3 times the standard deviation of the distributions.

The identification procedure relies on cuts on a likelihood function associated with each ring detected in the RICH-1 detector. The Likelihood function describes the signal number of photons taking into account the Frank and Tamm equation; the background number of photons, coming from other particles in the event and the beam halo, is evaluated from real data through the analysis of the photon detector occupancy.

Since particle identification with RICH is possible only above the Cherenkov threshold, a lower limit on hadron momentum has been applied: it is $\sim 3 \mathrm{GeV} / \mathrm{c}$ for $\pi$ and $\sim 10 \mathrm{GeV} / \mathrm{c}$ for $K$, values a little above the corresponding Cherenkov thresh- old in order to assure a minimum number of emitted photons. The upper limit for momentum has been chosen as $50 \mathrm{GeV} / \mathrm{c}$ for both $\pi$ and $K$, corresponding to $1.5 \sigma$ mass separation between the two hypothesis.

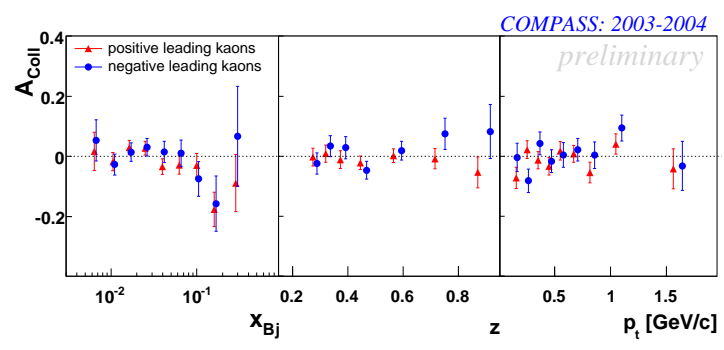

Figure 3. Collins asymmetries for positive (triangles) and negative (circles) leading $\mathrm{K}$ as a function of $x, z$ and $p_{T}$.

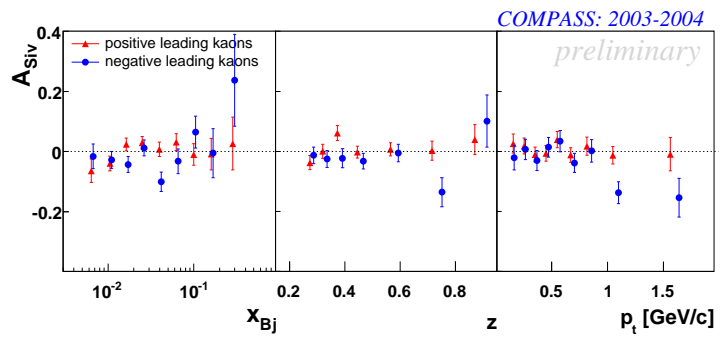

Figure 4. Sivers asymmetries for positive (triangles) and negative (circles) leading $\mathrm{K}$ as a function of $x, z$ and $p_{T}$.

After the identification, the final hadron sample consist of $5.2 \cdot 10^{6}$ and $4.5 \cdot 10^{6}$ positive and negative $\pi$, and $0.9 \cdot 10^{6}$ and $0.45 \cdot 10^{6}$ positive and negative $K$. The results on pion sample are very similar to those on unidentified hadrons (since more than $80 \%$ of the hadrons are pions). The 
results on $\mathrm{K}$ sample are shown in fig. 3 and 4 . also in this case, the asymmetries are very small and compatible with zero within the statistical errors.

\section{HADRON PAIR ASYMMETRIES}

A different probe of the transversity function is the hadron pair production.

The fragmentation function of a quark into a pair of charged hadrons is expected to exhibit an azimuthal dependence [10]-[13], so that the number of events can be written as:

$N=N_{0} \cdot\left(1+f \cdot P_{t} \cdot D_{n n} \cdot A_{\phi_{R S}} \cdot \sin \left(\phi_{R S}\right)\right)$

where $\phi_{R S}$ is defined as $\phi_{R}-\phi_{s^{\prime}}$. The $\phi_{R}$ angle is the angle between the lepton scattering plane and the plane containing the virtual photon momentum $\mathbf{q}$ and the component $R_{T}$ of the relative hadron momentum $\mathbf{R}=\frac{1}{2}\left(\mathbf{P}_{1}-\mathbf{P}_{2}\right)$ which is perpendicular to the summed hadron momentum $\mathbf{P}_{h}=\mathbf{P}_{1}+\mathbf{P}_{2}$. The asymmetry $A_{\phi_{R S}}$ is proportional to the transversity function convoluted with the fragmentation function describing the two hadrons production, $H_{1}^{L h}$ :

$A_{\phi_{R S}} \propto \frac{\sum_{q} e_{q}^{2} \Delta_{T} q(x) H_{1}^{L h}\left(z, M_{h}^{2}\right)}{\sum_{q} e_{q}^{2} q(x) D_{q}^{h}\left(z, M_{h}^{2}\right)}$

where $M_{h}^{2}$ is the invariant mass of the hadron pair and $z=z_{1}+z_{2}$.

The asymmetries have been measured on all the charged hadron pairs, for a total of $\sim 6.1 \cdot 10^{6}$ combinations. The results as a function of $x, M_{h}$ and $z$, are presented in fig. 5 . The errors shown are only the statistical contribution. The measured asymmetries are compatible with zero within the errors also in this case.

\section{CONCLUSIONS}

COMPASS is the first experiment probing transversity in DIS on a deuterium target. Our measured asymmetries are small and compatible with zero, within the measured errors, which typically are of the order of $1 \%$. Since the HERMES collaboration have measured non zero asymmetries using a proton target, the most probable interpretation for our data is a cancellation between

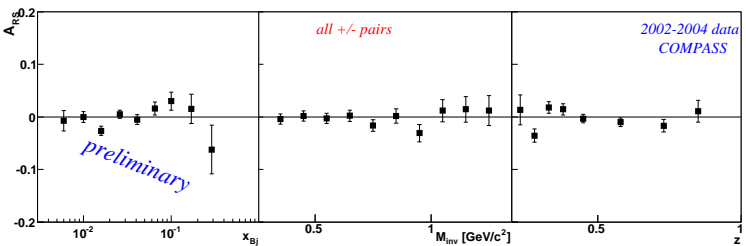

Figure 5. Asymmetries $A_{\phi_{R S}}$ for charged hadron pairs as a function of $x, M_{h}$ and $z$.

the contribution coming from the proton and the neutron.

COMPASS is planning to have a transversity data taking with a proton target in 2007 .

\section{REFERENCES}

1. R.L. Jaffe, X. Ji, Phys. Rev. Lett. 67, 552 (1991).

2. J. Collins, Nucl. Phys. B 396, 161 (1993).

3. D. Sivers, Phys. Rev. D 41, 83 (1990).

4. D. Boer, P.J. Mulders, Phys. Rev. D 57, 5780 (1998).

Phys. Rev. Lett. 94, 012002 (2005)

5. V. Y. Alexakhin et al. [COMPASS Collaboration], Phys. Rev. Lett. 94 (2005) 202002.

6. E. S. Ageev et al. [COMPASS Collaboration], Nucl. Phys. B 765, 31 (2007) arXiv:hep-ex/0610068.

7. G. Baum et al.[COMPASS collaboration], CERN-SPSLS-96-14;

8. G.K. Mallot, Nucl. Instrum. Meth. A 518, 121 (2004);

9. E. Albrecht et al., Nucl. Instrum. Meth. A 504, 2003 (354)

10. J.R. Collins, S.F. Heppemann and J.A. Ladinsky , Nucl. Phys. B 420, 565 (1994).

11. X. Artru and J.R. Collins, Z. Phys. C 69, 277 (1996).

12. M. Radici, R. Jacob, A. Bianconi, Phys. Rev. D 65, 074031 (2002).

13. A. Bacchetta and M. Radici, Phys. Rev. D 69, 074026 (2004). 